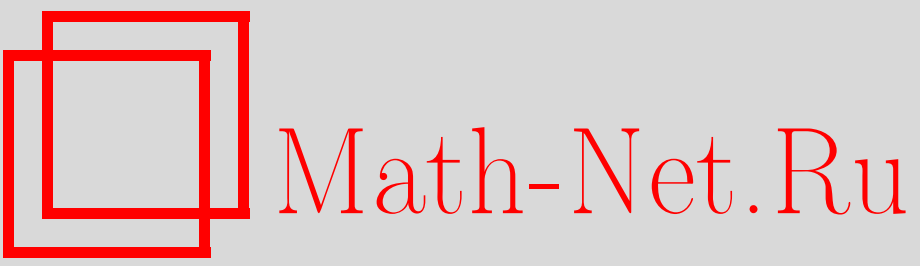

А. А. Феликсон, Кокстеровские разбиения сферических симплексов с неразрезанными двугранными углами, УМН, 2002, том 57, выпуск 2, 201-202

DOI: https://doi.org/10.4213/rm508

Использование Общероссийского математического портала Math-Net.Ru подразумевает, что вы прочитали и согласны с пользовательским соглашением

http://www.mathnet.ru/rus/agreement

Параметры загрузки:

IP: 3.89 .185 .249

26 апреля 2023 г., 12:59:04 


\title{
КОКСТЕРОВСКИЕ РАЗБИЕНИЯ СФЕРИЧЕСКИХ СИМПЛЕКСОВ С НЕРАЗРЕЗАННЫМИ ДВУГРАННЫМИ УГЛАМИ
}

\author{
А. А. ФЕЛикСОН
}

ОПРЕДЕЛЕниЕ. 1. Многогранник в сферическом пространстве $S^{n}$ назьвается многогранником Кокстера, если все его двугранные углы являются целыми частями $\pi$.

2. Кокстеровским разбиением выпуклого многогранника $P$ назьвается такое его разбиение на конечное число кокстеровских многогранников, что любые два многогранника разбиения, имеющие общую гипергрань, симметричны друг другу относительно нее.

3. Многогранники разбиения назьваются фундаментальными. Плоскость $\alpha$, содержащая гипергрань какого-нибудь фундаментального многогранника, называется зеркалом, если $\alpha$ не содержит гиперграни многогранника $P$.

4. Пусть задано кокстеровское разбиение многогранника $P$. Двугранньй угол многогранника $P$, образованньй гипергранями $\alpha$ и $\beta$, назьвается неразрезанныц, если $\alpha \cap \beta$ не принадлежит ни одному зеркалу.

5. Кокстеровское разбиение называется неразло жимым, если его фундаментальньй многогранник неразложим.

Несложно показать, что любое кокстеровское разбиение симплекса, имеющее разрезанньй двугранный угол, либо является в некотором определенном смысле композицией других кокстеровских разбиений, либо может быть получено с помощью алгоритма, описанного в [1]. В данной работе найдены все кокстеровские разбиения сферических симплексов с неразрезанными двугранными углами. Это позволяет закончить классификацию кокстеровских разбиений сферических симплексов, а также важно для описания кокстеровских разбиений гиперболических симплексов с неразрезанными двугранными углами.

Пусть задано кокстеровское разбиение с неразрезанньми двугранными углами выпуклого многогранника $P$ в $S^{n}$. Тогда $P$ - многогранник Кокстера и, следовательно, симплекс. Фундаментальный многогранник при этом также является симплексом. Несложно доказать следующую лемму:

Лемма 1. Пусть $E_{1}$ и $E_{2}-$ евклидовы пространства, а $E=E_{1} \oplus E_{2}$. Пусть $\Gamma_{i}-$ группа ортогональных преобразований пространства $E_{i}$, порожденная отражениями $(i=1,2), \Gamma=\Gamma_{1} \times \Gamma_{2}$ u $\Delta$ - подгруппа группь $\Gamma$, породсденная отражениями. Тогда $\Delta=\Delta_{1} \times \Delta_{2}$, где $\Delta_{i}-$ подгруппа группь $\Gamma_{i}$, порожденная отражениями $(i=1,2)$.

Все кокстеровские разбиения сферических симплексов являются прямыми произведениями неразложимых разбиений. Двугранные углы прямого произведения разбиений неразрезаны тогда и только тогда, когда неразрезаны двугранные углы сомножителей.

Будем обозначать через $F$ тип фундаментального многогранника кокстеровского разбиения. Для типов сферических симплексов Кокстера будут использованы стандартные обозначения $A_{n}$, $B_{n}, D_{n}, \ldots$.

Теорема 1. Пусть симплекс $P$ допускает неразлоэкимое кокстеровское разбиение $c$ неразрезанными двугранными углами и фундаментальным симплексом $F$. Тогда пара $(F, P)$ однозначно определяет кокстеровское разбиение. Все возможнные такие пары перечислены в таблице:

\begin{tabular}{|c|c||c|c|}
\hline$F$ & $P$ & $F$ & $P$ \\
\hline$H_{3}$ & $3 A_{1}$ & $E_{6}$ & $A_{5}+A_{1}, 3 A_{2}$ \\
\hline$F_{4}$ & $2 A_{2}$ & $E_{7}$ & $D_{6}+A_{1}, A_{5}+A_{2}, 2 A_{3}+A_{1}, A_{7}, D_{4}+3 A_{1}, 7 A_{1}$ \\
\hline$H_{4}$ & $A_{4}, 2 G_{2}^{(5)}, 2 A_{2}$, & \multirow{2}{*}{$E_{8}$} & $\begin{array}{c}A_{8}, A_{7}+A_{1}, A_{5}+A_{2}+A_{1}, 2 A_{4}, 4 A_{2}, A_{6}+A_{2}, A_{7}+A_{1}, \\
D_{8}, D_{6}+2 A_{1}, D_{5}+A_{3}, 2 D_{4}, D_{4}+4 A_{1}, 2 A_{3}+2 A_{1}, 8 A_{1}\end{array}$ \\
\hline & $H_{3}+A_{1}, D_{4}, 4 A_{1}$ & & $D_{m_{1}}+\cdots+D_{m_{r}}\left(m_{1}+\cdots+m_{r}=n\right)$, \\
& & $D_{n}$ & $m_{1} \geqslant m_{2} \geqslant \cdots \geqslant m_{r}>1,2 \partial e D_{2}=2 A_{1}, D_{3}=A_{3}$ \\
\hline
\end{tabular}


Для случаев, когда $F \neq H_{3}, H_{4}, F_{4}, B_{n}$, эта классификация непосредственно вытекает из результатов Е. Б. Дьнкина [2].

За исключением случаев $F=H_{3}$ и $F=H_{4}$, группе $\Gamma_{F}$, порожденной отражениями в гранях симплекса $F$, отвечает система корней $\Delta_{F}$. Группа $\Gamma_{P}$, порожденная отражениями в гранях симплекса Кокстера $P$, является подгруппой в $\Gamma_{F}$. Подгруппе $\Gamma_{P}$ отвечает система корней $\Delta_{P}$, являющаяся подсистемой в $\Delta_{F}$, если $F \neq B_{n}, F_{4}$. В [2] Е. Б. Дынкиным перечислены регулярные полупростые подалгебры алгебр Ли, или, что то же самое, перечислены подсистемы корней в системах корней. Таким образом, для доказательства теоремы достаточно: 1) среди регулярных подалгебр алгебр $A_{n}, D_{n} E_{6}, E_{7}, E_{8}$ выбрать подалгебры полного ранга; 2$)$ найти все кокстеровские разбиения с неразрезанными углами и фундаментальным симплексом типа $B_{n}, F_{4}, H_{3}$ или $H_{4}$.

Лемма 2. Пусть $(F, P)$ - кокстеровское разбиение с неразрезанными двугранными углами. Тогда для любой вериины $v$ схемь Кокстера $P$ существует такая вериина $w$ схемь $F$, что либо $P \backslash v=F \backslash w$, либо симплекс $p$, соответствующий подсхеме $P \backslash v$, допускает кокстеровское разбиение с неразрезанными углами и фундаментальным симплексом $f$, соответствующим подсхеме $F \backslash w$.

Лемму несложно доказать, рассмотрев малую сферу с центром в вершине симплекса $P$.

ДокаЗАтЕльство теоРемы 1 . Рассмотрим $S^{n}$ как сферу единичного радиуса в евклидовом пространстве $\mathbb{E}^{n+1}$ с ортонормированным базисом $e_{1}, \ldots, e_{n+1}$. Тогда симплексу $F$ соответствует $n$-гранный конус. Пусть в случаях $B_{n}$ и $F_{4}$ множество $\Delta$ (система корней) состоит из векторов $\pm e_{i}, \pm e_{i} \pm e_{j}$ и $\pm e_{i}, \pm e_{i} \pm e_{j}, \frac{1}{2}\left( \pm e_{1} \pm e_{2} \pm e_{3} \pm e_{4}\right)$ соответственно. В случае $F=H_{4}\left(H_{3}\right)$ выберем координаты так, чтобы единичными нормалями к граням конуса были векторы $(0,-1,0,0), \frac{1}{4}(2, \alpha, \beta, 0),(-1,0,0,0), \frac{1}{4}(2,0, \alpha, \beta)$, где $\alpha=1+\sqrt{5}, \beta=1-\sqrt{5}$. Тогда множество $\Delta$ нормалей к зеркалам отражений группы, порожденной отражениями относительно граней конуса, - это все векторы, получающиеся четными перестановками координат векторов $(1,0,0,0), \frac{1}{2}( \pm 1, \pm 1, \pm 1, \pm 1)$ и $\frac{1}{4}(2, \pm \alpha, \pm \beta, 0)$. (Случаю $H_{3}$ соответствуют те вектора, в которых последняя координата равна нулю.)

Пусть $F=B_{n}$. Из табл. 9 работы [2] следует, что $P$ содержит прямое слагаемое $D_{n}(n>1$, $\left.D_{3}=A_{3}, D_{2}=2 A_{1}\right)$. Можно считать, что $D_{n}$ содержит векторы $e_{1} \pm e_{2}$ и, посколюку $e_{2} \in M$, $P$ имеет разрезанный двугранный угол.

Пусть $F=F_{4}, H_{3}$ или $H_{4}$. Из леммы 2 следует, что если $(F, P)$ не совпадает ни с одним разбиением из условия теоремы 1 , то $F=H_{4}, P=A_{2}+G_{2}^{(5)}, A_{3}+A_{1}, 2 A_{1}+A_{2}$ или $2 A_{1}+G_{2}^{(5)}$. Таких разбиений нет, поскольку в $\Delta$ нет соответствующей четверки векторов. Для каждого из разбиений, перечисленных в условии, несложно найти векторы из $\Delta$, перпендикулярные граням симплекса $P$. В случае разбиения $\left(F_{4}, 2 A_{2}\right)$ выберем векторы $e_{1}-e_{2}, e_{2}-e_{3},-\frac{1}{2}\left(e_{1}+e_{2}+e_{3}+e_{4}\right)$, $e_{4}$. Легко проверить, что при этом нет разрезанных двугранных углов. Остальные разбиения не могут иметь разрезанных двугранных углов, поскольку симплексы $H_{3}$ и $H_{4}$ не имеют двугранных углов, равных $\pi / 4$.

То, что неразложимое разбиение однозначно определяется парой $(F, P)$, в случаях $F=E_{6}$, $E_{7}, E_{8}$ и $D_{n}$ следует из [1], а в каждом из осталнных случаев - из того, что по лемме 2 симплекс $P$ имеет такую вершину $v$, что грани фундаментальных симплексов, содержащих $v$, однозначно определены с точностью до симметрии симплекса $P$.

Автор благодарит Э.Б. Винберга за поддержку и постоянное внимание к работе.

\section{СПИСОК ЛИТЕРАТУРЫ}

[1] A. Felikson // European J. Combin. 1998. V. 19. P. 801-817. [2] Е. Б. Дынкин // Матем. сб. 1952. Т. 30. № 2. С. 349-462.

Московский государственньй

Принято редколлегией университет им. М. В. Ломоносова 